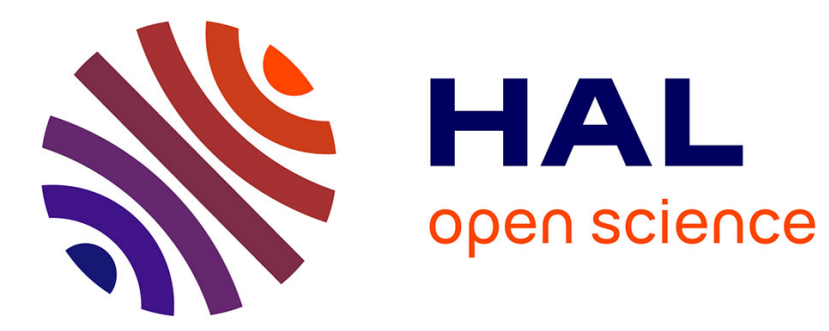

\title{
Préparation d'échantillons de carbone pour sources d'ions à pulvérisations
}

\author{
I. Brissaud, J.P. Mouffron, J.M. Garnier, J.L. Michelot
}

\section{To cite this version:}

I. Brissaud, J.P. Mouffron, J.M. Garnier, J.L. Michelot. Préparation d'échantillons de carbone pour sources d'ions à pulvérisations. Revue de Physique Appliquée, 1983, 18 (8), pp.519-522. 10.1051/rphysap:01983001808051900 . jpa-00245114

\section{HAL Id: jpa-00245114 https://hal.science/jpa-00245114}

Submitted on 1 Jan 1983

HAL is a multi-disciplinary open access archive for the deposit and dissemination of scientific research documents, whether they are published or not. The documents may come from teaching and research institutions in France or abroad, or from public or private research centers.
L'archive ouverte pluridisciplinaire HAL, est destinée au dépôt et à la diffusion de documents scientifiques de niveau recherche, publiés ou non, émanant des établissements d'enseignement et de recherche français ou étrangers, des laboratoires publics ou privés. 


\title{
Préparation d'échantillons de carbone pour sources d'ions à pulvérisations
}

\author{
I. Brissaud, J. P. Mouffron \\ Institut de Physique Nucléaire, B.P. 1, 91406 Orsay Cedex, France \\ J. M. Garnier et J. L. Michelot \\ Laboratoire d'Hydrologie et Géochimie Isotopique, \\ Bât. 504, Université Paris-Sud, 91405 Orsay, France
}

(Reçu le 14 janvier 1983, révisé le 8 avril, accepté le 18 avril 1983)

\begin{abstract}
Résumé. - Des échantillons de carbone ont été préparés par craquage d'acétylène dans une décharge radiofréquence. Dans une source d'ions négatifs d'un accélérateur tandem, les dépôts permettent d'obtenir un faisceau de ${ }^{12} \mathrm{C}^{-}$aussi intense qu'avec du graphite pur. Le fractionnement isotopique ${ }^{13} \mathrm{C} /{ }^{12} \mathrm{C}$ introduit par le craquage a été mesuré. Les résultats montrent que ces échantillons de carbone peuvent être utilisés pour des expériences de datation par le ${ }^{14} \mathrm{C}$ sur les accélérateurs tandem.
\end{abstract}

Abstract. - Numerous carbon samples were prepared by RF cracking of acetylene. These samples give a ${ }^{12} \mathrm{C}^{-}$ current as intense as pure graphite when used in a sputtering ion source of tandem accelerator. Isotopic fractionation ${ }^{13} \mathrm{C} /{ }^{12} \mathrm{C}$ is measured.

Les sources d'ions d'accélérateur de particules doivent, dans de nombreuses expériences, délivrer des faisceaux ioniques d'une grande intensité pour que le nombre d'événements recherchés soit significatif et la durée de l'expérience raisonnable.

Dans le cas où un accélérateur est utilisé comme spectromètre de masse [1], cette condition est impérative car il s'agit d'accélérer un isotope peu abondant. Pour une datation par le carbone 14 , on accélère un faisceau de cet isotope contenu dans l'échantillon naturel. L'échantillon à dater (carbonate, charbon de bois, tourbe, os, etc...) doit donc subir un traitement chimique et physique pour aboutir à une forme chimique sous laquelle les isotopes du carbone représentent la quasi-totalité de la prise d'essai. De cette façon, le rendement de source est optimisé. Pour ce type d'expérience, nous disposons d'un accélérateur tandem muni d'une source d'ions négatifs à pulvérisation inversée. Dans celle-ci, un faisceau primaire de césium de quelques $\mathrm{keV}$ vient frapper le carbone, déposé au fond d'un puits percé dans un petit cylindre d'aluminium, et arrache les ions $\mathrm{C}^{-}$qu'une électrode extrait avant l'injection dans l'accélérateur. Le graphite est la forme cristalline du carbone qui donne un courant d'ions maximum; mais il est difficile de donner cette forme cristalline au carbone d'un échan- tillon naturel. Aussi, convient-il de réaliser une procédure simple de préparation du carbone telle que la source délivre un faisceau ionique d'une intensité proche de celle du graphite.

\section{Fabrication d'un dépôt de carbone pur.}

Un certain nombre d'études ont été faites par Beukens et al. [2] et Grootes et al. [3] sur le craquage de l'acétylène dans une décharge radiofréquence. Devant la relative simplicité de cette technique, nous l'avons adoptée pour la préparation de carbone destiné au tandem d'Orsay.

L'acétylène peut être obtenu à partir d'un échantillon naturel selon une procédure bien connue [4] : l'attaque acide ou la combustion de l'échantillon produit du dioxide de carbone qui réagit à chaud avec du lithium pour donner un carbure; l'hydrolyse de ce carbure fournit l'acétylène. Notre dispositif (Fig. 1) de craquage comprend une enceinte cylindrique de $14 \mathrm{~cm}$ de haut et $4 \mathrm{~cm}$ de diamètre contenant le gaz. Deux cylindres, porteurs d'échantillons de la source, servent d'électrodes pour l'application du champ radiofréquence; ils sont percés d'un puits d'un diamètre de $2 \mathrm{~mm}$ et d'une profondeur de $0,5 \mathrm{~mm}$ logeant le dépôt. 


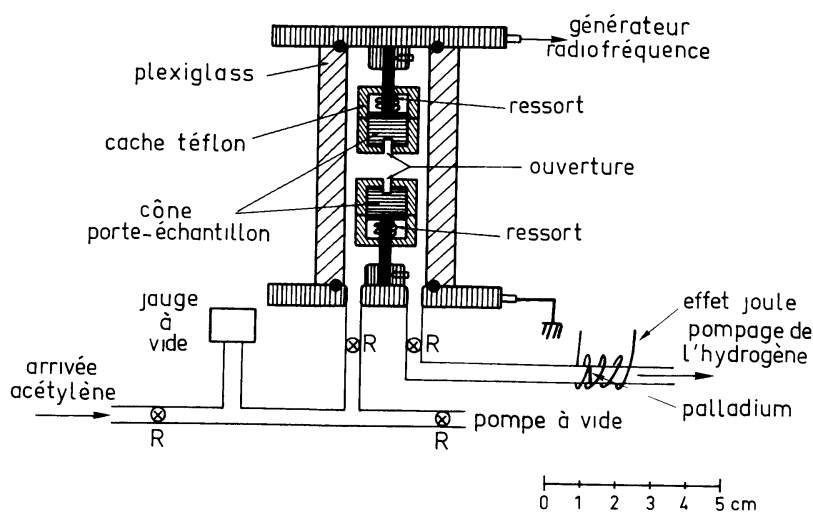

Fig. 1. - Schéma de l'enceinte de craquage de l'acétylène. [Scheme of a chamber of acetylene cracking.]

Un cache en téflon permet de limiter la surface du dépôt. La distance entre les deux cylindres, de l'ordre de $10 \mathrm{~mm}$, est un paramètre peu important. La décharge a lieu entre les cylindres et un plasma se développe : le carbone issu de la décomposition de l'acétylène se dépose de façon symétrique dans les deux puits. La forme de la décharge et sa couleur sont dépendantes de façon sensible de la pression du gaz : à une pression inférieure à 5 torrs, cette décharge est d'un aspect mal défini, de couleur violette; le dépôt brunâtre qui en résulte est fragile. Les parois de l'enceinte se couvrent d'un voile de carbone. Par contre, à une pression supérieure à 15 torrs, la décharge de couleur verdâtre est filiforme, de diamètre très inférieur à celui des puits, et ses extrémités mobiles à l'intérieur de ceux-ci.

Entre ces deux situations, la décharge de couleur bleue est spatialement bien définie d'un orifice à l'autre des caches. Le dépôt s'effectue avec seulement quelques projections sur les bords des puits. Dans ces dernières conditions lors du craquage, la pression gazeuse reste constante, indice d'une simple décomposition stoechiométrique conservant les volumes. Par contre, si le craquage s'effectue à pression inférieure à 5 torrs, celle-ci chute rapidement du fait de la formation de polymères. Pour connaître en permanence la pression d'acétylène, nous avons pompé l'hydrogène produit à travers une feuille de palladium de $0,1 \mathrm{~mm}$ chauffée à environ $500{ }^{\circ} \mathrm{C}$. Mais, le rendement de cette fuite est assez faible et l'on se propose d'augmenter celui-ci par l'utilisation d'un cylindre de palladium d'une surface plus grande.

Dans les conditions expérimentales retenues, le craquage est effectué à une pression de 10 torrs; au bout de $5 \mathrm{~min}$. le gaz résiduel est pompé. En renouvelant l'opération 5 fois, on obtient un dépôt total de $1,7 \mathrm{mg}$ (soit $70 \mu \mathrm{g} \mathrm{min.}^{-1}$ ) par cylindre, largement suffisant pour les pesées et les études de faisceau d'ions. Le procédé par radiofréquence est plus rapide d'un facteur 7 que la décomposition par une décharge continue [5]. Le rendement exprimé par le rapport. du poids de carbone déposé sur les deux électrodes au poids initial de carbone de l'acétylène dans l'enceinte, est proche de $65 \%$.

Le carbone ainsi obtenu a été étudié par diffraction de rayons $X$. On ne remarque aucune structure cristalline contrairement au graphite; cette structure amorphe est comparable à celle des noirs de carbone. A l'oil, le dépôt présente une surface au relief mameloné (Fig. 2); il est peu fragile et résiste assez bien aux chocs et manipulations.
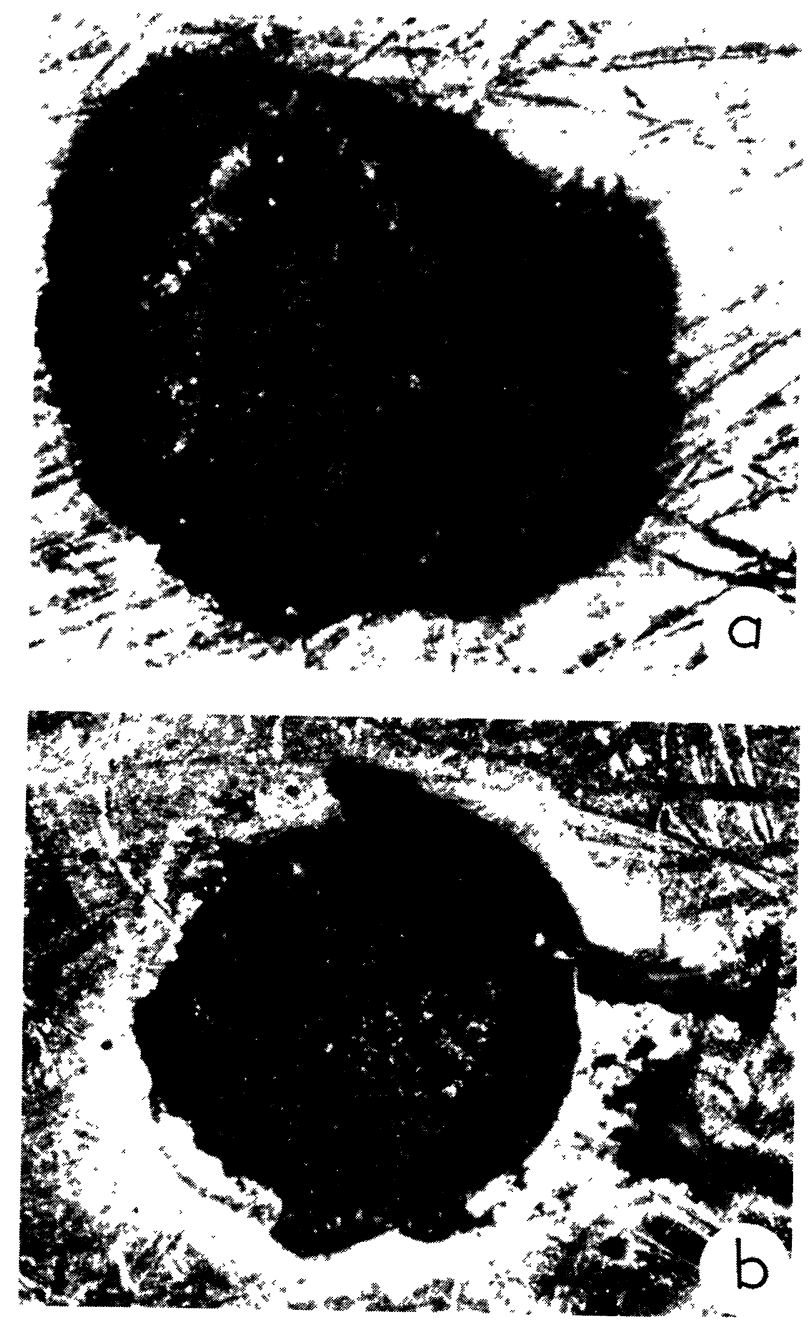

Fig. 2. - Photographie du dépôt de carbone avant (a) et après $(b)$ l'utilisation dans la source d'ions. Le diamètre et la profondeur du trou sont respectivement de 2 et $0,5 \mathrm{~mm}$.

[View of a carbon deposit before $(a)$ and after $(b)$ use in the ion source.]

\section{Caractéristiques du faisceau d'ions carbone.}

Les dépôts réalisés selon la procédure décrite ont été étudiés sur le banc d'essais de l'Institut de Physique Nucléaire d'Orsay. Ce banc comprend une source d'ions Genionex, identique à celle du tandem, accouplée à un spectromètre magnétique. Une cage de 
Faraday située dans le plan image mesure le courant ionique en fonction du champ magnétique. Par balayage de ce dernier, on obtient le spectre de particules émises par la source. A l'aide de ce banc, nous avons comparé l'évolution du courant ionique de ${ }^{12} \mathrm{C}^{-}$d'un dépôt de craquage et d'un échantillon de graphite pur servant de référence. La figure 3 montre que les intensités sont dans les deux cas pratiquement égales et voisines de $6 \mu \mathrm{A}$. L'émission ionique atteint son maximum après $50 \mathrm{~min}$., puis reste approximativement constante durant plusieurs heures (un essai réalisé sur $14 \mathrm{~h}$ aurait pu être prolongé). Cette stabilité signifie que la spectrométrie de masse des isotopes du carbone est réalisable sans difficulté, puisque la durée de telles mesures est de l'ordre de l'heure [1]. L'intensité du faisceau de césium est approximativement $0,2 \mathrm{~mA}$. Si on augmente fortement ce courant (d'un ordre de grandeur), l'intensité du faisceau de $\mathrm{C}^{-}$ croît également, mais la durée de vie du dépôt en est fortement réduite. Ces résultats très satisfaisants permettent d'envisager l'adoption de la technique de craquage dans la fabrication d'échantillons de carbone à partir d'échantillons naturels.

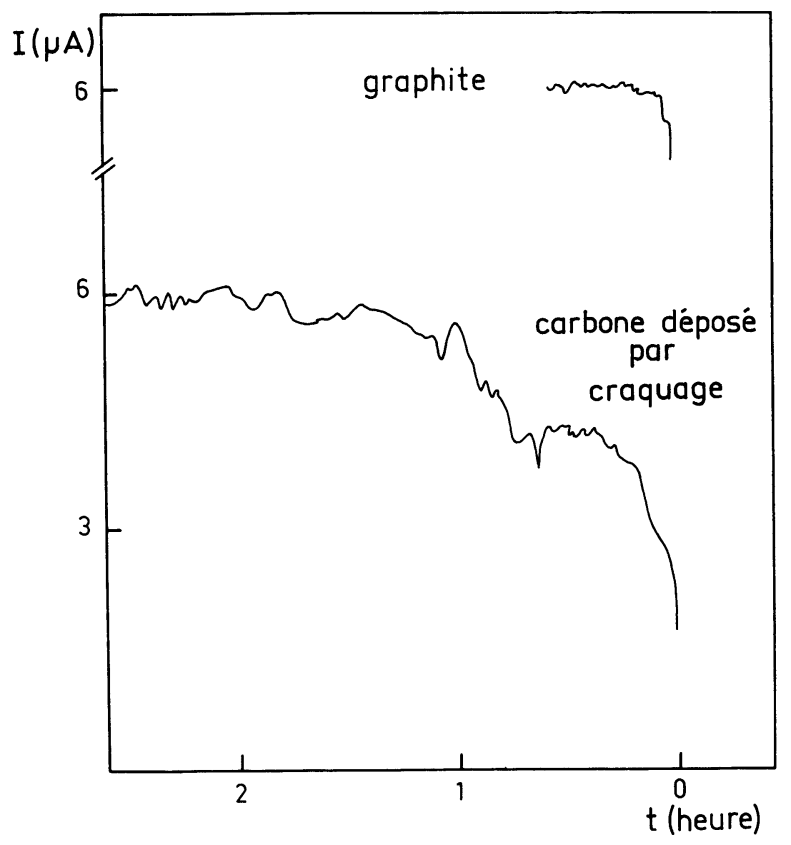

Fig. 3. - Enregistrement expérimental donnant, en fonction du temps, l'intensité d'ions $\mathbf{C}^{-}$émis par une source à pulvérisation à partir de graphite pur ou de carbone déposé par craquage d'acétylène.

${ }^{12} \mathrm{C}^{-}$ions intensity for a sputtering pure graphite of cracked carbon.]

Différents essais supplémentaires ont été effectués de façon à tester l'effet de certains facteurs :

- pression d'acétylène : sous une pression de 5 torrs, le craquage donne un dépôt à partir duquel la source délivre un faisceau d'ions d'une intensité réduite d'un facteur 5 . Avec une pression de 20 torrs, l'intensité est également faible ( $\cong 100 \mathrm{nA})$ et le dépôt se détériore très vite sous l'action du bombardement de césium;

- épaisseur du dépôt : c'est un facteur important pour assurer une émission ionique constante et prolongée. Ainsi la même quantité de carbone déposée dans un puits de $5 \mathrm{~mm}$ de diamètre, au lieu de $2 \mathrm{~mm}$ précédemment, produit un courant dont l'intensité chute d'un facteur 2 ;

- profondeur du puits : elle ne doit pas être trop grande car, en passant d'une profondeur de $0,5 \mathrm{~mm}$ à $2 \mathrm{~mm}$, l'intensité est réduite d'un facteur 4 pour une même épaisseur du dépôt ;

- générateur radio-fréquence : le modèle utilisé délivre une tension variable atteignant $20000 \mathrm{~V}$ avec une fréquence de $100 \mathrm{kHz}$ et une puissance maximum de $30 \mathrm{~W}$. Divers essais réalisés en faisant varier la tension ne montrent aucune différence sensible sur la rapidité et le rendement du craquage.

La grandeur de l'intensité est liée à des effets de surface du dépôt de carbone que le faisceau de césium érode et chauffe lors de son premier essai sur le banc de source. Après un arrêt, le temps nécessaire pour atteindre l'intensité maximum est plus court que la première fois et cela d'autant plus que l'interruption est brève, donc que la température a peu baissé. En plus de la montée en température, produite par le bombardement de césium, l'émission ionique a lieu par transmission à travers la couche de césium qui se dépose sur le carbone [6]. Si une telle couche a déjà été déposée au cours d'une précédente émission, une nouvelle ionisation peut être rapidement réalisée une fois la montée en température atteinte. Les figures $2 a$ et $2 b$ montrent la photographie du dépôt avant et après la pulvérisation. On voit que la surface du dépôt n'a pas été bouleversée, mais simplement émoussée, alors que l'émission avait duré plus de $14 \mathrm{~h}$ avec une intensité voisine de $6 \mu \mathrm{A}$. Si l'on continue l'émission, un cratère se forme peu à peu.

Une très fine pellicule de carbone, très fragile au toucher, est parvenue à se fixer à l'extérieur et autour des trous malgré les caches (Fig. $2 a$ ). Sous l'action du faisceau de césium, cette couche a disparu très rapidement (Fig. 2b). Cette attaque superficielle est, du reste, utilisée pour nettoyer le dépôt de carbone et éliminer ainsi toute contamination éventuelle de la surface. Dans l'exemple choisi, le dépôt pèse $1,6 \mathrm{mg}$. Après le bombardement de $14 \mathrm{~h}$, le poids a légèrement varié ; il n'est que d'environ $1,2 \mathrm{mg}$. Si environ $40 \mu \mathrm{g}$ ont été reçus sous forme d'ions négatifs par la cage de Faraday, une grande partie du carbone manquant a été projetée sur les parois, où apparaît un léger voile brunâtre; ce sont les ions positifs et neutres qui ont été recueillis. Mais les essais ont montré que la principale perte de matériau se produit par pulvérisation de fragments. L'importance de ces dommages est très aléatoire. 


\section{Fractionnement isotopique.}

Les échantillons préparés selon cette procédure peuvent servir à des expériences de datation par le ${ }^{14} \mathrm{C}$ à l'aide des accélérateurs tandem. Elles consistent à mesurer ${ }^{14} \mathrm{C} /{ }^{12} \mathrm{C}$ mais il faut connaître, pour la détermination de ce rapport, le fractionnement introduit par la préparation de l'échantillon (il faut aussi connaître le fractionnement isotopique produit par l'émission dans la source). Le fractionnement qui affecte ${ }^{14} \mathrm{C}$ peut être évalué à partir du fractionnement sur le ${ }^{13} \mathrm{C}$ par la relation [7] :

$$
{ }^{14} \mathrm{C} /{ }^{12} \mathrm{C}=2,3{ }^{13} \mathrm{C} /{ }^{12} \mathrm{C} \text {. }
$$

Le rapport ${ }^{13} \mathrm{C} /{ }^{12} \mathrm{C}$ est mesuré usuellement par un spectromètre de masse à double collecteurs. Une étude antérieure [8] a montré que le fractionnement, introduit dans la confection de l'acétylène à partir d'un échantillon naturel, n'est que de $0,3 \%$ en valeur absolue. Nous avons mesuré le rapport ${ }^{13} \mathrm{C} /{ }^{12} \mathrm{C}$ dans des échantillons préparés par craquage rapide (le volume d'ącétylène est renouvelé après $1 \mathrm{~min}$.) et dans les échantillons préparés avec un temps de craquage plus long (procédure normale), l'écart entre les deux rapports est de $0,022 \%$ et la moyenne de $1,084 \%$. Si l'on compare cette dernière valeur à celle de l'acétylène $(1,0867 \pm 0,022 \%)$, on constate que le craquage n'a entraîné qu'un léger appauvrissement en ${ }^{13} \mathrm{C} d u$ dépôt de carbone malgré une décomposition incomplète.

Dans une molécule, la fréquence de vibration d'un atome augmente si la masse de celui-ci diminue; c'est-à-dire que pour l'acétylène, la fréquence est plus grande pour le carbone 12 que pour le carbone 13 . Dans la décharge radiofréquence, l'énergie fournie augmente ces vibrations jusqu'à la rupture des liaisons électroniques. Celle-ci affecte donc préférentiellement les molécules constituées des isotopes les plus légers [9].

\section{Remerciements.}

Nous remercions $Y$. Kœechlin pour de nombreuses discussions ainsi que A. Filly, P. Lecolle, J. F. Saliege pour les mesures de fractionnement isotopique et que F. Planche pour les macrophotographies.

\section{Bibliographie}

[1] Purser, K., Litherland, A. E. and Gove, H. E., Nucl. Instrum. Methods 162 (1979) 637.

[2] Beukens, R. P., et al., Atomic Energy of Canada, Report TR 39 (1980).

[3] Grootes, P. M. et al., Radiocarbon 22 (1980) 487.

[4] TAMers, M. A., J. Appl. Radiat. Isotopes 26 (1975) 676.

[5] Sznajderman, D. et Izac, J., Communication privée.
[6] Kilius, L. R., Ph. D. Thesis, Toronto, Canada (1980) non publiée.

[7] SAlièGe, J. F., soumis à J. Appl. Radiat. Isotopes (1983).

[8] Garnier, J. M., Thèse 3e cycle, Université Paris VI (1976).

[9] Genet, M., Communication privée. 